\title{
Desarrollo reproductivo de Krapfia weberbaueri (Ranunculaceae) en condiciones controladas de luz y temperatura
}

\section{Reproductive development of Krapfia weberbaueri (Ranunculaceae) under controlled conditions of light and temperature}

\author{
Beatriz Roca ${ }^{1,2}$, Mery Suni ${ }^{1,3}$, Asunción Cano ${ }^{1,3}$
}

1 Laboratorio de Fisiología Vegetal. Facultad de Ciencias Biológicas, Universidad Nacional Mayor de San Marcos. Dirección Postal 11-0058. Lima 11, Perú

2 Laboratorio de Florística, Departamento de Dicotiledóneas, Museo de Historia Natural, Universidad Nacional Mayor de San Marcos.

3 Instituto de Investigación de Ciencias Biológicas Antonio Raimondi (ICBAR), Facultad de Ciencias Biológicas, UNMSM.

Email Beatriz Roca: beatrizrocaramos@yahoo.com

Email Mery Suni: msunin@gmail.com

Email Asunción Cano: ashuco@yahoo.com

Citación:

Roca B., M. Suni, A. Cano. 2014. Desarrollo reproductivo de Krapfia weberbaueri (Ranunculaceae) en condiciones controladas de luz y temperatura. Rev. peru. biol. 20(3): 233 - 240 (Marzo 2014)

\section{Resumen}

Krapfia weberbaueri Standl. \& J. F. Macbr. es una especie endémica del Perú, categorizada como vulnerable y distribuida en los departamentos de Ancash, Huánuco, Junín y San Martín. El presente trabajo reporta las observaciones sobre el desarrollo reproductivo de esta especie bajo condiciones controladas de luz y temperatura ( 12 horas de luz/12 horas de oscuridad y $12{ }^{\circ} \mathrm{C}$ día y $8{ }^{\circ} \mathrm{C}$ noche). Plantas completas en estado reproductivo fueron recolectadas en la Cordillera Blanca (Ancash) donde forma parches entre los 4200 y $4800 \mathrm{~m}$ de altitud. Los datos morfométricos de los órganos florales fueron registrados cada dos o tres días con un vernier y se evaluó la producción de néctar a lo largo del desarrollo de la flor. Asimismo se evaluó la viabilidad de los granos de polen y la receptividad del estigma. Krapfia weberbaueri tiene flores muy llamativas de 24 a $47 \mathrm{~mm}$ de longitud, que desde la antesis hasta el inicio de senescencia se mantienen receptivas aunque en mayor medida en la flor madura, etapa en la cual la dehiscencia de estambres y liberación de los granos de polen alcanza el 100\%. Este periodo suma un total de 13 días. La producción del néctar se inicia en la flor madura y continua hasta la senescencia total de los pétalos. La viabilidad de los granos de polen varió de 78 a $97 \%$ y el éxito de producción de aquenios fue 38 a $62 \%$. Se puede señalar que la polinización es favorecida por la posición final de las anteras sobre los estigmas, producción del néctar, moderada presencia y desplazamiento de ácaros y áfidos y la posición pendular de la flor.

Palabras clave: Perú; Ancash; floración; ginóforo; néctar.

\section{Abstract}

Krapfia weberbaueri Standl. \& J. F. Macbr. is an endemic species from Peru, categorized as vulnerable, and distributed in the departments of Ancash, Huanuco, Junin and San Martin. This work reports the reproductive development of the species under controlled conditions of light and temperature (12 hours light/12 hours darkness, $12{ }^{\circ} \mathrm{C}$ during light hrs and $8{ }^{\circ} \mathrm{C}$ during dark hrs). Entire plants at reproductive stage were collected at Cordillera Blanca (Ancash), where it is found forming patches, at an elevation of 4200 to $4800 \mathrm{~m}$. The species has large and showy flowers, which from anthesis to the onset of senescence remain responsive, however at a greater extent in the mature flower, stage at which the dehiscence of stamens and release of pollen grains reaches $100 \%$. This period lasts a total of 13 days. Nectar production begins when the flower matures and continues until the total senescence of petals. The viability of the pollen grains varied from 78 to $97 \%$ and success in achene production was $38-62 \%$. It may be noted that pollination is favored by the final position of anthers over the stigmas, nectar production, moderate presence and movement of mites and aphids, and the pendulous position of the flower.

Keywords: Peru; Ancash; flowering; gynophore; nectar. 


\section{Introducción}

La familia Ranunculaceae está representada por alrededor de 50 géneros y más de 2000 especies, con amplia distribución, desde hierbas perennes o anuales, terrestres, acuáticas o semiacuáticas (Tamura 1993). En el Perú, la familia Ranunculaceae está representada por ocho géneros y 50 especies (Brako \& Zarucchi 1993; Ulloa et al. 2004), en su mayoría hierbas. Uno de los géneros presentes en el Perú y endémico de los Andes de Sudamérica es Krapfia DC, que se distribuye encima de los 3500 $\mathrm{m}$ de altitud en Colombia, Ecuador, Perú y Bolivia (Lourteig 1956). Actualmente se reconoce nueve especies para el género (Lourteig 1956, Tamura 1993). Las especies de este género presentes en el territorio peruano habitan principalmente laderas rocosas con fuerte pendiente de zonas húmedas. Se caracterizan por presentar flores globosas que no se abren por completo durante su desarrollo, sépalos persistentes generalmente rojizos externamente y un ginóforo bien desarrollado (Lourteig 1956, Tamura 1993).

Krapfia weberbaueri Standl. \& J. F. Macb. es una especie perenne, endémica del Perú y categorizada como vulnerable según los criterios de la UICN (León et al. 2007); se distribuye en los departamentos de Ancash, Huánuco, Junín y San Martin (Macbride 1937). Tiene flores grandes y muy llamativas, por lo que es utilizada como planta ornamental, así como también sus flores son usadas en medicina tradicional andina, por la creencia de que ellas propician el desarrollo del lenguaje en los niños que tienen dificultad en el habla; ambos motivos serían los causantes de una fuerte depredación por el hombre (información obtenida en las zonas de estudio). El presente trabajo describe el desarrollo y características florales de Krapfia weberbaueri, en base a observaciones en laboratorio bajo condiciones controladas de temperatura e iluminación, lo que permite conocer aspectos básicos de su biología y usados en su conservación.

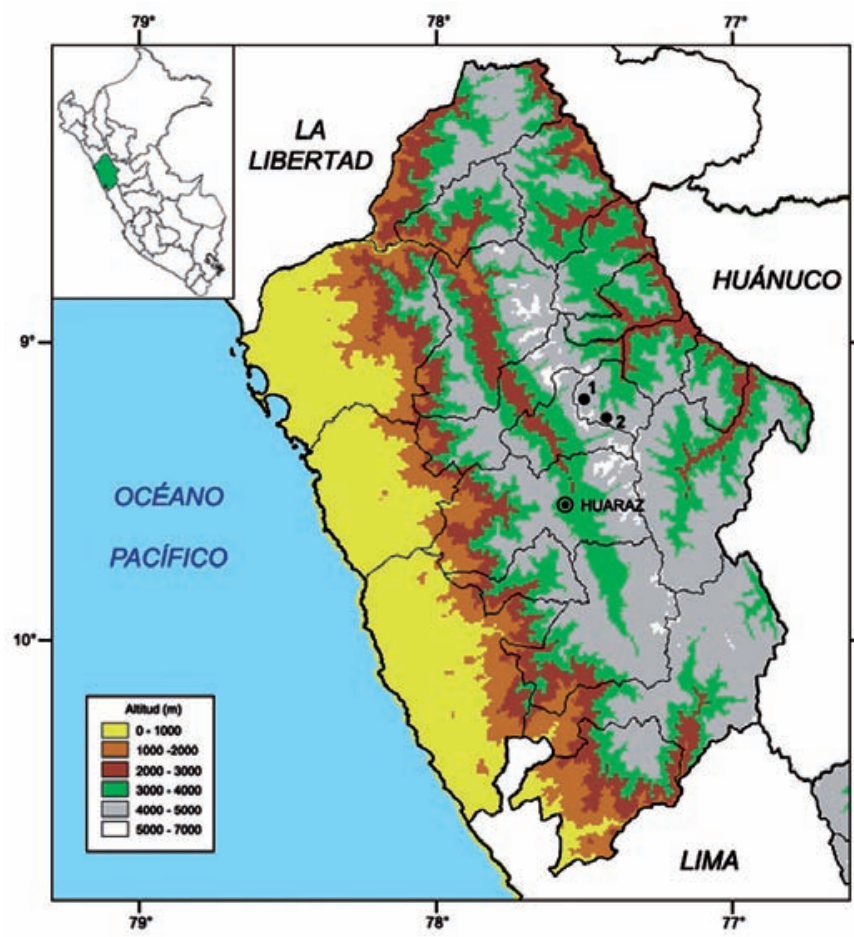

Figura 1. Puntos de muestreo de Krapfia weberbaueri. 1) cerca de la Laguna Librón (4800 m de altitud, 09³8.36'S, 077³8.36'W), 2) cerca de la Laguna Atlante (4700 m de altitud, 09¹5'12.19"S, $\left.077^{\circ} 26^{\prime} 24.068^{\prime \prime} \mathrm{W}\right)$.

\section{Material y métodos}

Material biológico.- Se recolectaron plantas completas en estado reproductivo (con yemas florales que presentaban tonalidad rojiza) en el distrito de Chacas, provincia de Asunción (Ancash) en el flanco oriental de la Cordillera Blanca, en lo que se conoce como Callejón de Conchucos. La primera colecta se realizó en la zona de Huichganga, situado arriba de la laguna Librón a pocos kilómetros del nevado Copa, a $4800 \mathrm{~m}$ de altitud (13 de Agosto 2008 , tres plantas). La segunda y tercera colecta se realizaron en la misma zona cerca de la laguna Atlante (18 de mayo, cuatro plantas y el 29 de septiembre de 2009, once plantas Figura 1).

Un total de 18 plantas con su respectivo sustrato fueron recolectadas y trasladadas en canastas hasta el Laboratorio de Fisiología Vegetal de la Facultad de Ciencias Biológicas de la Universidad Nacional Mayor de San Marcos, Lima, donde fueron colocadas en dos recipientes de $0.05 \mathrm{~m}^{2}$ y dentro de una cámara climática (VISION VS-3DS) con fotoperiodo de 12/12 horas de luz/oscuridad y termoperíodo de $12 / 8^{\circ} \mathrm{C}$ día/noche (similar a las temperaturas registradas in situ al momento de la colecta).

Cambios en el desarrollo floral.- Se realizaron observaciones y mediciones cada dos o tres días de todas las (18) flores en estudio, utilizando un vernier digital $(0.01 \mathrm{~mm})$. En cada fecha se determinaron:

- Longitud de la flor (medido desde la base del perianto, sin contar el pedúnculo, hasta el extremo distal del perianto o del gineceo si esta sobresale).

- Diámetro de apertura.

- Momento de la producción de néctar.

- Momento de la apertura de antera

- Momento de la liberación de granos de polen.

- La receptividad del estigma (entendida como la capacidad del estigma para sostener la germinación del polen) fue evaluada en todos los pistilos de cada flor en estadio de antesis, de flor abierta, flor madura y en inicio de senescencia usando peróxido de hidrógeno al 3\% (método de Osborn et al. 1988). El peróxido de hidrógeno era aplicado sobre los estigmas de los pistilos con la ayuda de una pipeta Pasteur, iniciando con los ubicados en la base y terminando con los del ápice del gineceo; se evaluaba la intensidad de la reacción observando la formación de burbujas.

- La fertilización efectiva se determinó por la relación entre los promedios del número de frutos por flor y el número de pistilos por flor.

- La viabilidad de los granos de polen se evaluó por la tinción con orceína y carmín acético 1\% (D'Ambrogio de Argüeso 1970), para esto se colectaron los granos de polen de las flores totalmente abiertas (flor madura) con dehiscencia y liberación de los granos de polen al cien por ciento, se colectó la mayor cantidad posible de granos de polen con la ayuda de un hisopo de algodón y fueron depositados en un vial de vidrio al que se agregó el colorante y se dejó por 24 horas en refrigeración. Se tomó una submuestra en una lámina porta objeto que contenía una gota de glicerina líquida al $50 \%$ y se procedió a la evaluación. Las lecturas se realizaron en más de 

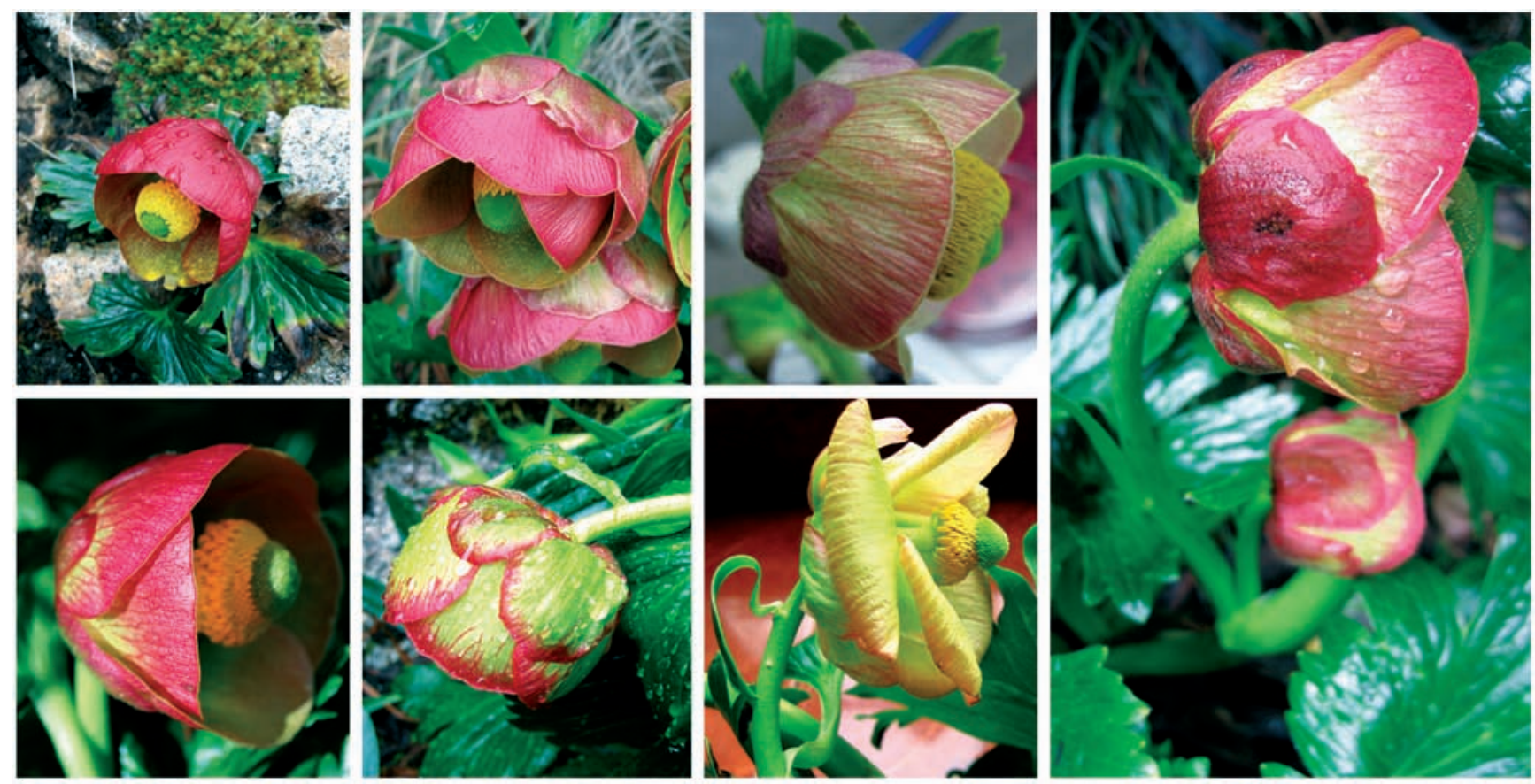

Figura 2. Variabilidad del color de los pétalos de las flores de Krapfia weberbaueri, observadas en lugar de colecta.

50 campos con la ayuda del microscopio óptico con un aumento de 400x, los granos de polen viables se tornan de una coloración rojiza y los no viables se mantienen de un color amarillo, adicionalmente se tomaron las dimensiones de los dos tipos de polen.

La viabilidad de los granos de polen así como los datos morfométricos florales fueron evaluados en las muestras obtenidas en las dos primeras colectas, mientras que con la tercera colecta se evaluó la receptividad del estigma.

Para validación de las diferencias entre las medidas se utilizó el análisis de varianza de una vía (ANOVA), el nivel de significación se consideró 0.05 .

\section{Resultados}

En el periodo de estudio se hicieron viajes en abril, mayo, agosto y septiembre a los hábitats de K. weberbaueri observando siempre plantas en floración por lo que se podría señalar que florece durante todo el año, aunque durante los meses de abril y mayo había mayor número de plantas en floración, también se observó que el perianto de las flores maduras presentaban variabilidad de tonalidades, que se mostraban en tonalidades entre verde amarillento a fucsia muy intenso. Así mismo, cada planta reproductiva presentaba generalmente una sola flor o yema floral, muy raras veces se observaron plantas con dos flores (Fig. 2).

Características de la flor y desarrollo floral de $K$. weberbaueri. - Al inicio del estudio las yemas florales tuvieron una

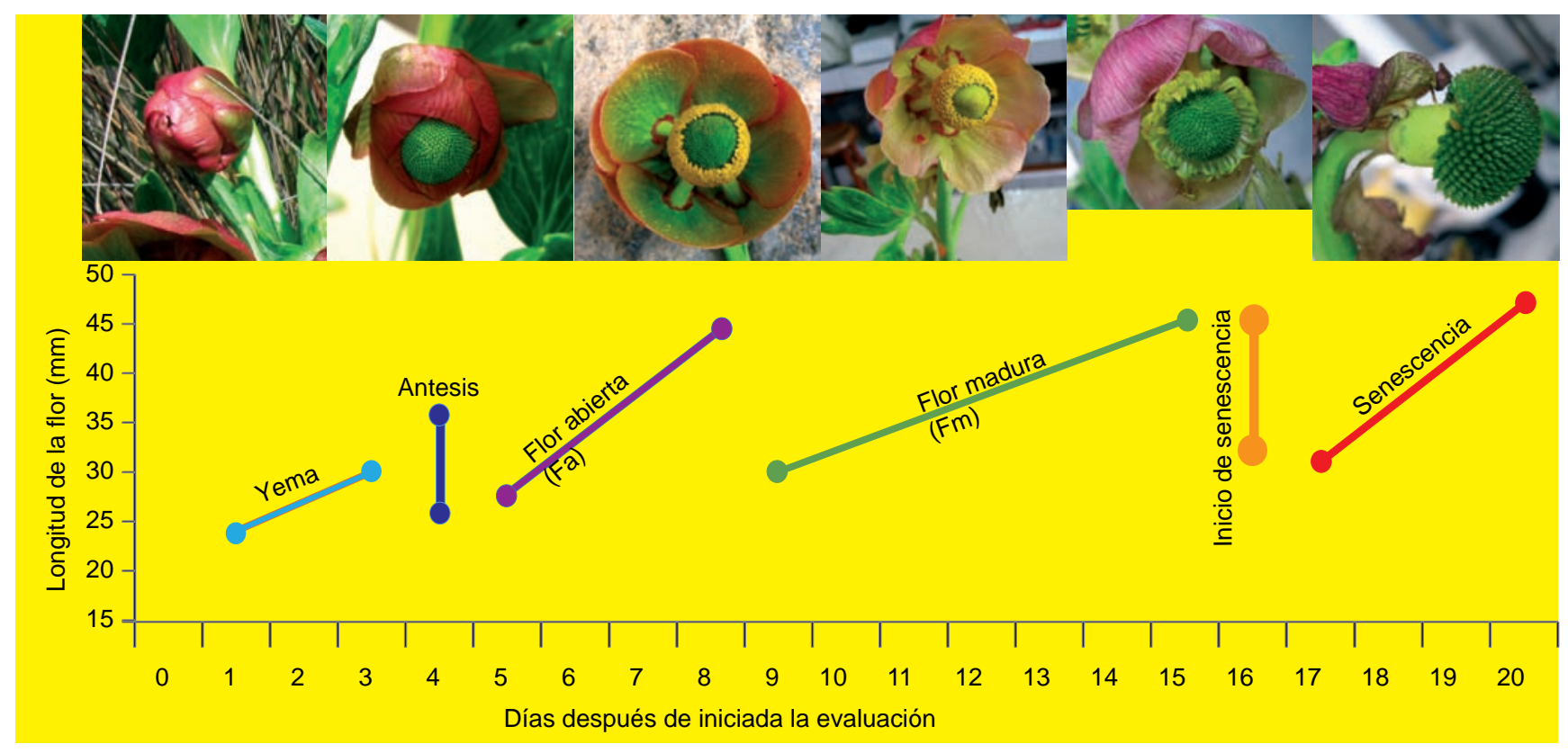

Figura 3. Desarrollo floral de Krapfia weberbaueri. Cada segmento indica la variación de la longitud de la flor y la duración en cada estadio. 

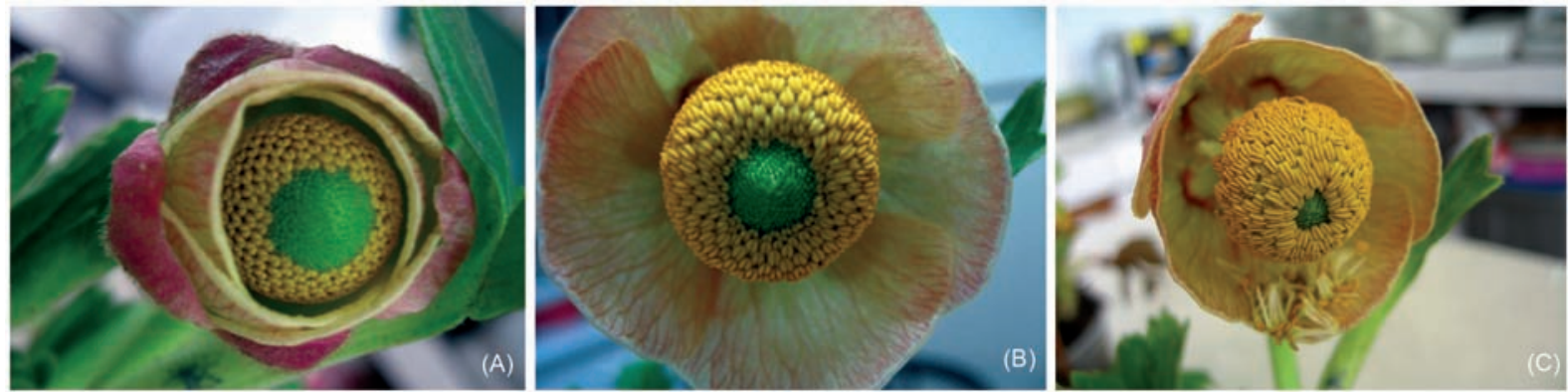

Figura 4. Encubrimiento de las anteras sobre el gineceo, desde la etapa de flor en antesis (A), flor abierta (B) y flor madura (C) con dehiscencia de las anteras y liberación de los granos de polen.

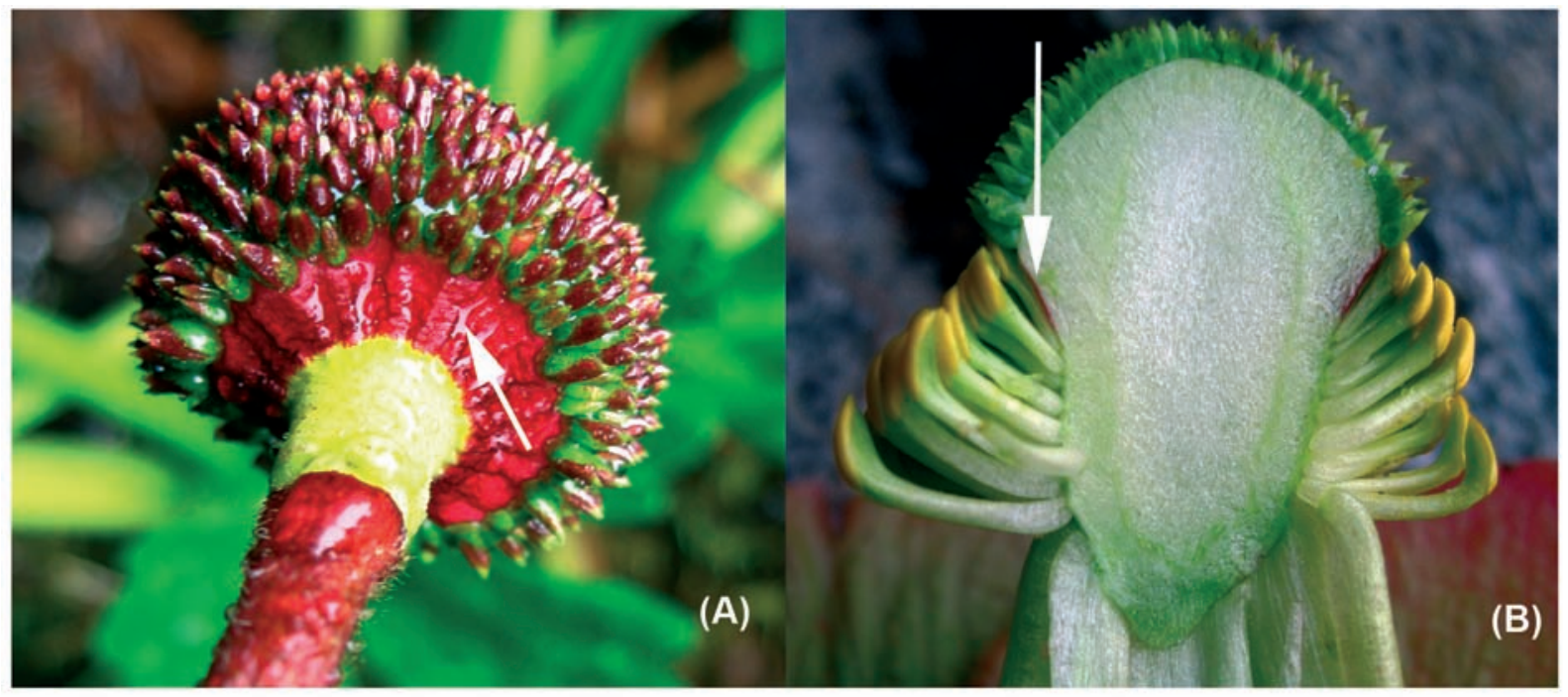

Figura 5. Vista superficial del ginóforo (A) y en sección longitudinal (B) en una flor en maduración. Glándulas nectaríferas se ubican en la base cuneada de la cara interna de cada pétalo, detrás y hacia la base de las escamas de color rojo intenso (Figura 6). Estas producen néctar a partir de la etapa de flor madura hasta inicio de flor senescente. El periodo de producción del néctar coincide con receptividad de estigma que dura 4 días.
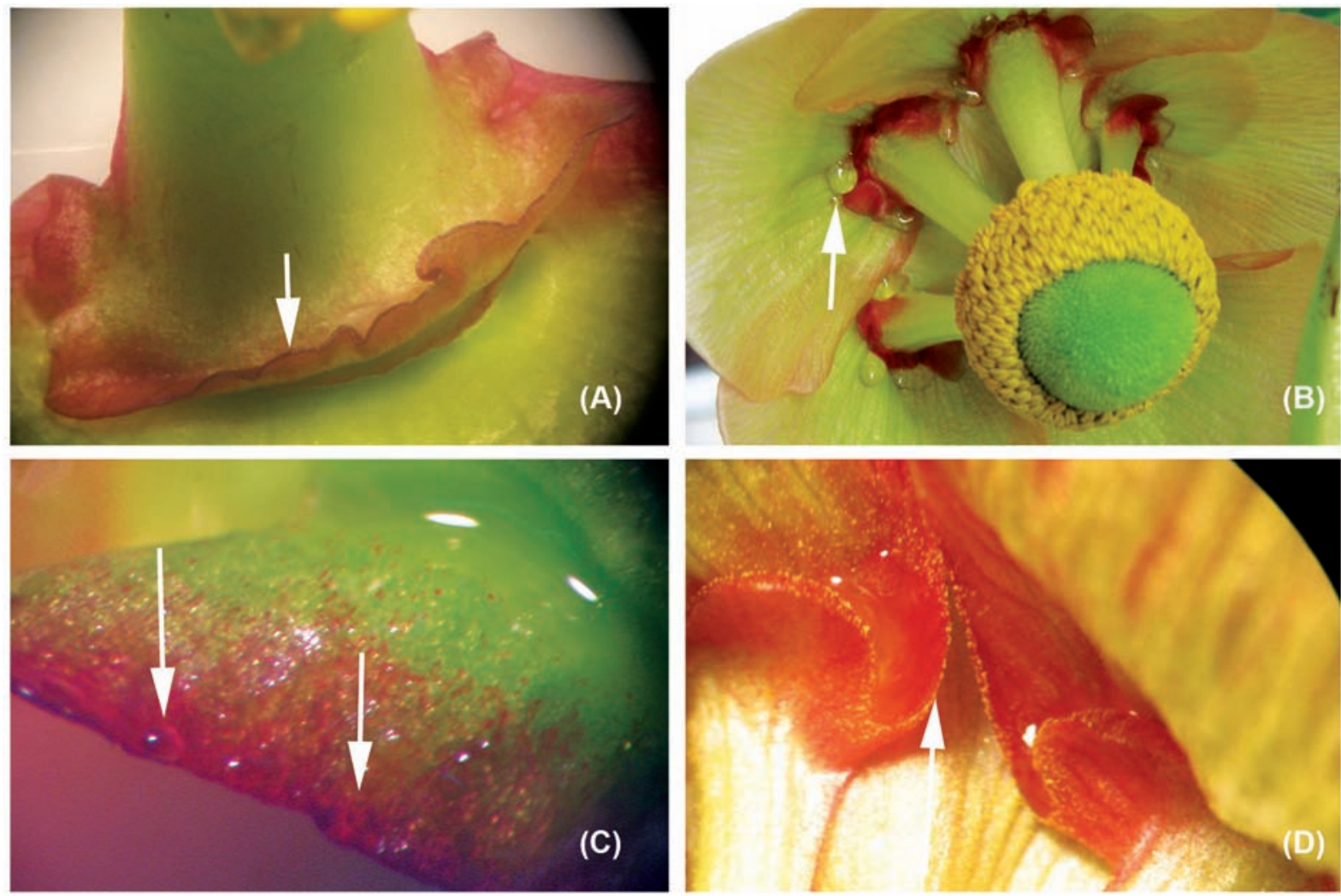

Figura 6. Detalles de escamas en la base cuneada de los pétalos (A -flecha), se observa la producción del néctar en la etapa de flor madura (B) las glándulas nectaríferas, ver flecha (C) y lugar donde se deposita el néctar (D). 


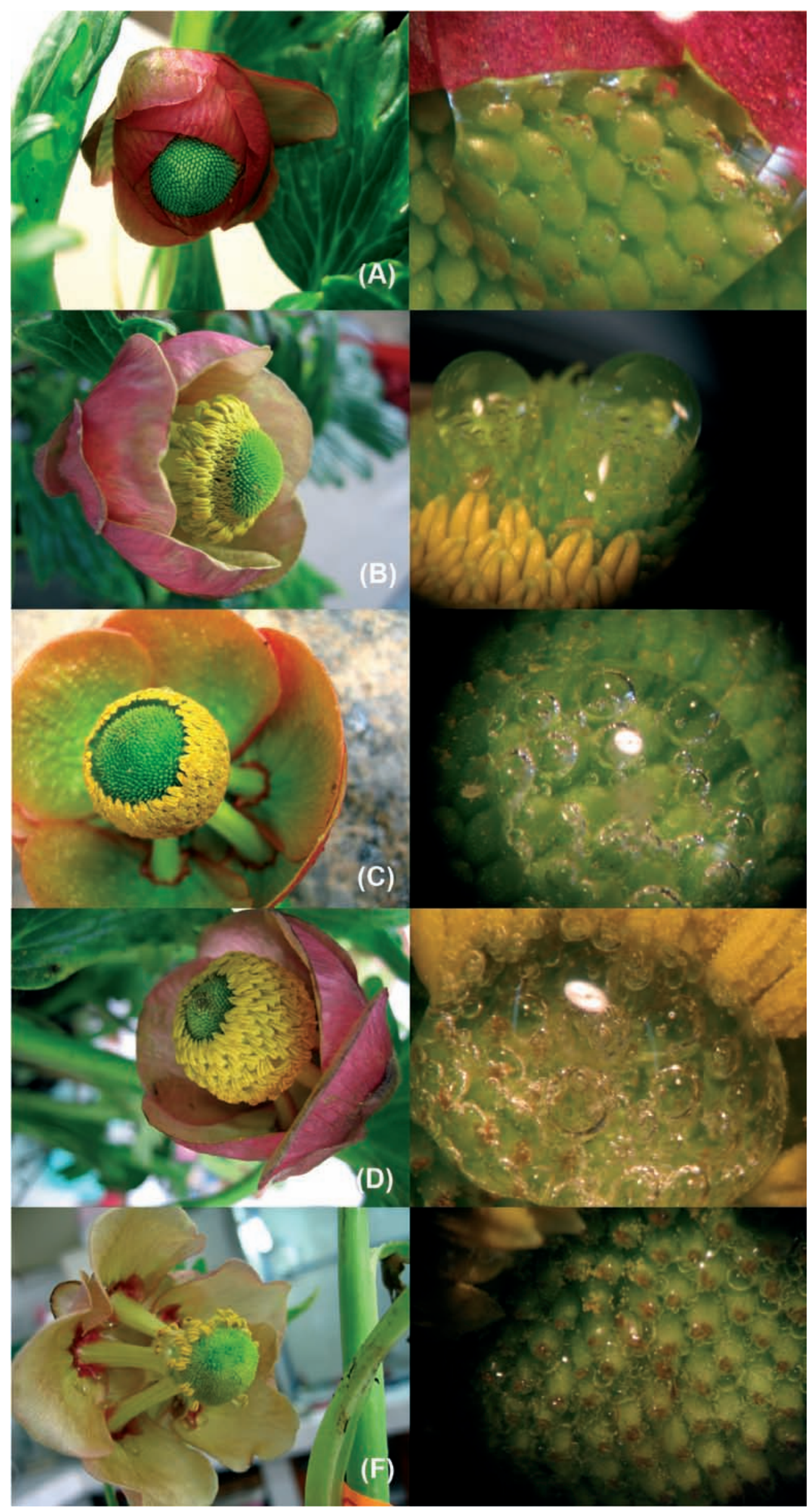

Figura 7. Receptividad del estigma evaluado con $\mathrm{H}_{2} \mathrm{O}_{2}$ en las diferentes etapas de desarrollo floral: (A) Flor en antesis (B), flor abierta, (C) flor madura, (D) flor en inicio de senescencia y (E) flor senescente, de Krapfia weberbaueri. 
longitud entre 24 y $32 \mathrm{~mm}$, y que se incrementaron hasta 47 $\mathrm{mm}$ al final del estudio. El proceso de desarrollo, desde antesis a inicio de la dispersión de frutos, se completó en 21 días, siendo 13 días el periodo entre la antesis y el inicio de senescencia floral (Fig. 3).

Se observó el desplazamiento de las anteras hacia el ápice cubriendo la superficie de la mayoría de los pistilos, paralelo con la dehiscencia de las anteras y la liberación de los granos de polen (Fig. 4). En la flor con perianto totalmente abierto, la longitud de la teca fue de $2.9 \mathrm{~mm}$ y del filamento $5.9 \mathrm{~mm}$.

Las flores presentaron más de 100 anteras de color amarillo intenso y pistilo verde oscuro, este último en algunos casos presento un color rojo intenso en la parte apical y verde en la parte basal. El ginóforo presentaba color rojo intenso y en corte longitudinal de la flor se observó como una zona libre entre la región estaminal y carpelar (Fig. 5).

Receptividad del estigma y liberación de los granos de polen.- La receptividad del estigma en el estadio de flor en antesis fue coincidió con el momento en que se inició la dehiscencia de las anteras y liberación de los granos de polen en los estambres ubicados en la base del androceo. En cambio, la receptividad se incrementó en la flor abierta, siendo máxima en estadio de flor madura (con mayor diámetro de apertura floral) coincidiendo con la total dehiscencia longitudinal de los estambres y la máxima liberación de los granos de polen. En el periodo de flor madura los filamentos de las anteras se elongaron y cubrieron la mayoría de los estigmas del gineceo mientras que en el inicio de la senescencia disminuyo la receptividad del estigma. Además, se observó que los pistilos que no llegaron a desarrollar aquenios (óvulos abortivos) todavía reaccionaban al peróxido de hidrógeno indicando que presentaban receptividad (Fig. 7 y 8 ).

La dehiscencia y la liberación de los granos de polen de los estambres ubicados en la base del androceo se inició en la etapa de flor en antesis, cuando sucede también la elongación de los estambres sobre la gran mayoría de los estigmas, lo que se incrementa en la flor madura. En la flor madura se produce la dehiscencia y liberación total de los granos de polen de todos los estambres.

En la flor madura pudimos observar abundantes ácaros y áfidos que se desplazaban por el gineceo dispersando grandes cantidades de granos de polen que portan en su cuerpo (Figs. 9 A, B , C y D).
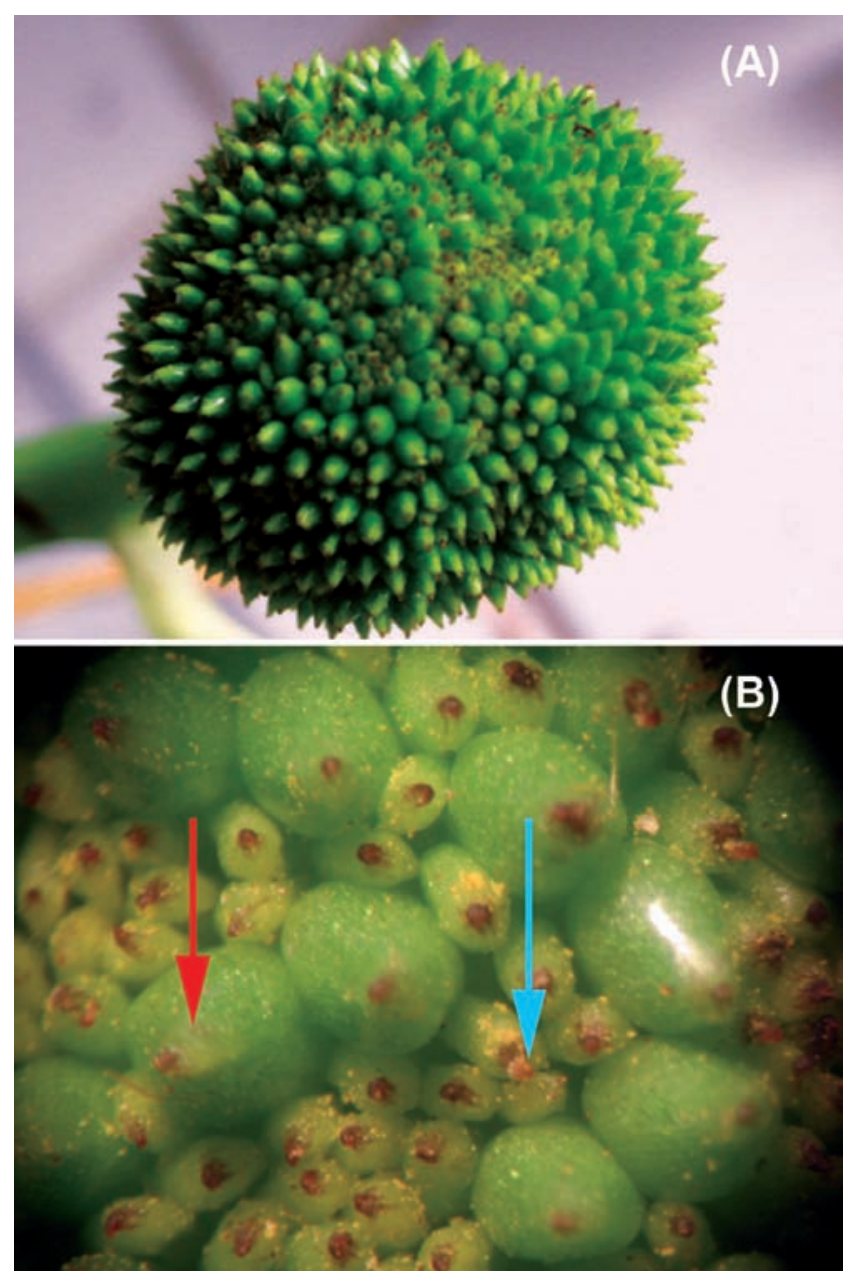

Figura 8. Formación de los aquenios (A). En (B) detalle de aquellos con desarrollo normal (flecha rojo) y aquenios abortivos (flecha celeste).

La viabilidad de los granos de polen fue la siguiente, en agosto 2008, los granos fértiles tuvieron $37.7 \mu \mathrm{m}$ de diámetro en promedio con $78 \%$ de viabilidad y para granos no fértiles 36 $\mu \mathrm{m}$ de diámetro. Mientras para mayo 2009, los granos fértiles tuvieron $37.75 \mu \mathrm{m}$ de diámetro con $97 \%$ de viabilidad y los no fértiles con $30.3 \mu \mathrm{m}$ de diámetro.

Al comparar los diámetros de los granos de polen de 2008 y 2009 , no se encontró diferencias significativas para los granos fértiles (ANOVA), pero sí para los granos no fértiles del 2009 (con diámetro menor) lo cual podría tener relación con el mayor

Tabla 1. Variables evaluadas durante el estudio del desarrollo reproductivo de Krapfia weberbaueri

\begin{tabular}{|c|c|c|c|c|}
\hline & & \multicolumn{2}{|c|}{ Colecta } & \multirow[b]{2}{*}{ Sig $0.05 \%$. } \\
\hline & & 2008 agosto & 2009 mayo & \\
\hline \multicolumn{2}{|l|}{$\mathrm{N}^{\circ}$ de pistilos/flor } & $442 \pm 33.33$ & $1015 \pm 5.07$ & $* *$ \\
\hline \multicolumn{2}{|l|}{$\mathrm{N}^{\circ}$ aquenios/flor } & $280 \pm 10.80$ & $769 \pm 9.22$ & $* *$ \\
\hline \multicolumn{2}{|c|}{ Fertilización efectiva \% } & 64.8 & 75.7 & ns \\
\hline \multicolumn{2}{|c|}{$\mathrm{N}^{\circ}$ de estambres/flor } & $300 \pm 34.71$ & $345 \pm 16.35$ & ns \\
\hline \multirow{5}{*}{ Granos de polen } & Long estambre mm & $8.9 \pm 0.19$ & $9.6 \pm 0.14$ & $* *$ \\
\hline & Diámetro fértiles $\mu \mathrm{m}$ & $37.7 \pm 0.69$ & $37.75 \pm 0.57$ & ns \\
\hline & Diámetro no fertiles $\mu \mathrm{m}$ & $35.95 \pm 0.90$ & $30.3 \pm 0.94$ & $* *$ \\
\hline & Fértiles \% $\left(\mathrm{N}^{\circ}\right)$ & $78(2208)$ & $96.7(4042)$ & $* *$ \\
\hline & No Fértiles \% $\left(\mathrm{N}^{\circ}\right)$ & $22(622)$ & $3.3(136)$ & $* *$ \\
\hline
\end{tabular}



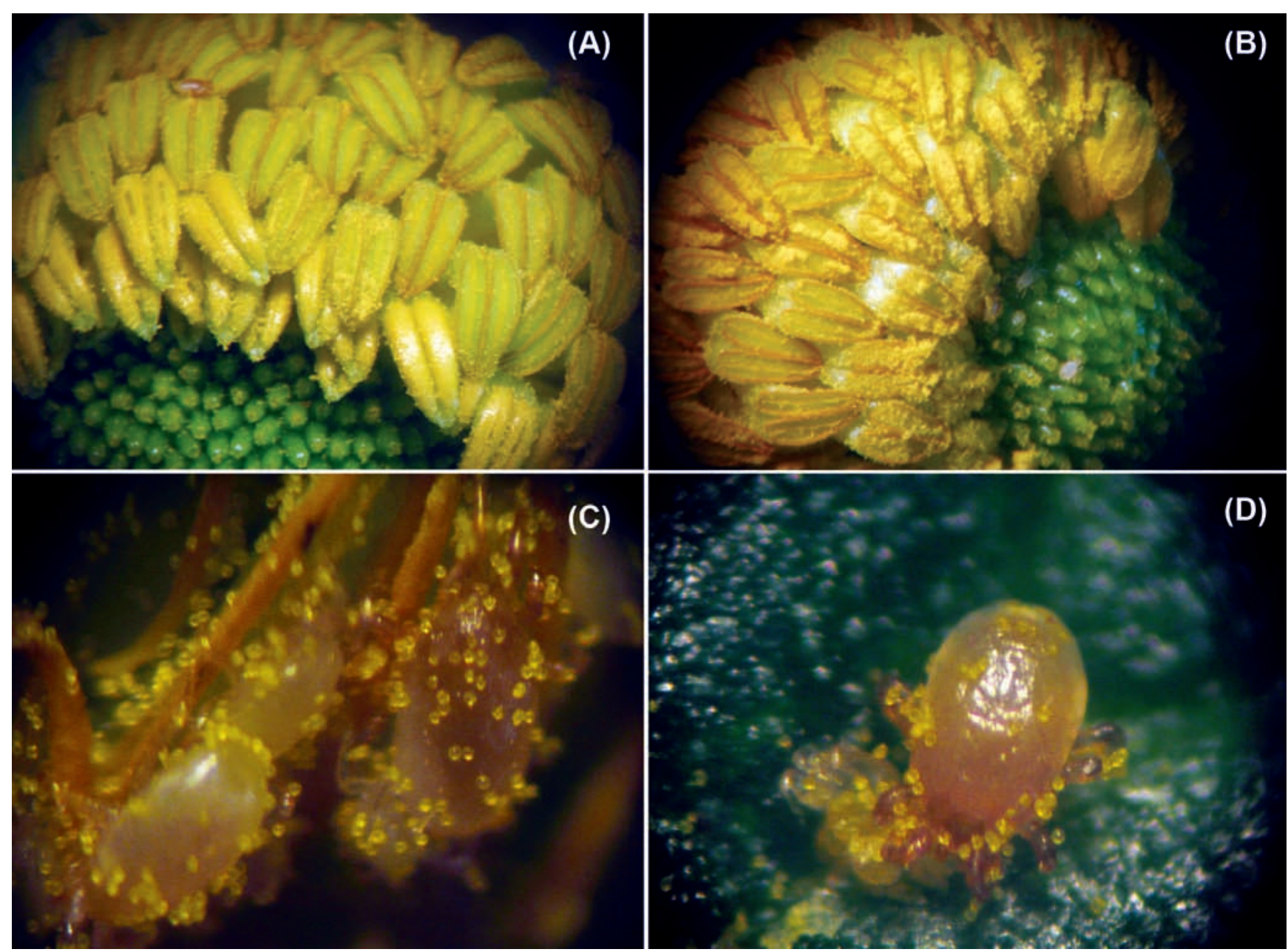

Figura 9. Los estambres con dehiscencia (A) y liberación de los granos de polen (B). Se incluye vista ampliada de presencia de ácaros (C y D).

porcentaje de los granos fértiles para ese año lo cual afectaría el tamaño de los no fértiles.

En las muestra recolectada en agosto 2008, el número promedio de estambres por flor fue de 300 , con longitud promedio de $8.9 \mathrm{~mm}$, y en las muestras de mayo 2009, se tuvo un número promedio de 345 estambres por flor, con longitud promedio de $9.6 \mathrm{~mm}$. Una observación adicional realizada en septiembre 2009, cuando las condiciones climáticas mejoraban, determino un promedio de 323 estambres por flor con 8.9 mm longitud en promedio (Tabla 1). Las anteras fueron de color amarillo intenso, bitecadas, basifijas con dehiscencia longitudinal.

El gineceo es apocárpico con pistilos de color verde oscuro, arreglados sobre el ginóforo. El ovario es unilocular y uniovular que termina en un estigma sésil. Se encontró para agosto 2008 un promedio de 442 pistilos por flor. Con pistilos de longitud y diámetro en promedio (a nivel del ovario) de los siguiente, flor en antesis $1.6 \times 0.5 \mathrm{~mm}$, en madura de $2.6 \times 1.1 \mathrm{~mm}$ y flor en senescencia de 2.7 x $1.5 \mathrm{~mm}$. En mayo de 2009 las flores tenían un promedio de 1015 pistilos, las diferencias entre las dos colectas eran estadísticamente significativas.

Fruto es un aquenio de color verde oscuro a guinda que se dispersa apenas senescen los pétalos. En agosto 2008 los aquenios tuvieron en promedio de $2.7 \times 1.5 \mathrm{~mm}$ de diámetro y el número promedio de aquenios por flor y por planta fue 280 (los restantes fueron abortivas), por lo que la fertilización efectiva fue de $63.3 \%$. En mayo de 2009 el número de aquenios por flor y planta fue de 769 aquenios equivalente al $75.8 \%$ de fertilización efectiva.

En general los valores para las variables evaluadas fueron mayores para mayo 2009 excepto para la variable diámetro de grano de polen fértil que no presenta diferencia significativa.

\section{Discusión}

Las diferencias encontradas para la mayoría de los caracteres florales entre las dos fechas evaluadas (Tabla 1), mayo 2009 (término del periodo de lluvias) y agosto de 2008 (estación seca sin precipitaciones), podrían estar más relacionados con las diferencias en temperatura y humedad en dichas fechas, que al efecto de la altitud señalado por Cursach y Rita (2012). También, en general las condiciones climáticas tendrían un mayor efecto sobre el desarrollo floral y el éxito reproductivo de $K$. weberbaueri, más que las condiciones de fertilidad y de contenido de agua del suelo como ocurre con otras especies (Timmerman-Erskine \& Boyd 1999).

Cabe resaltar que, aunque los caracteres $\mathrm{N}^{\circ}$ de pistilos/flor y $\mathrm{N}^{\circ}$ de frutos/flor muestran diferencias significativas entre las fechas, la fertilización efectiva no es significativamente diferente, lo cual podría indicar condiciones de estabilidad de su hábitat.

Se ha señalado que las especies bajo condiciones extremas como las altoandinas muestran principalmente mecanismos de autopolinización aunque también invierten buena proporción 
de su energía en la producción de néctar y grandes cantidades de polen destinados a la atracción de los polinizadores lo cual podría disminuir mucho el recurso energético para la fecundación de sus óvulos (Arroyo 1988). En nuestro estudio encontramos casos de autopolinización lo que ocurren cuando se desplaza el androceo sobre el gineceo durante el periodo de maduración y liberación de granos de polen. Además, el hecho de que las flores de $K$. weberbaueri son de gran tamaño, con colores intensos y con buena cantidad de polen y néctar, favorece la presencia de ácaros y áfidos en la planta (Torres et al. 2007) quienes, con su desplazamiento dentro de la flor, donde son frecuentes y de moderada abundancia, cumplirían el rol de polinizadores pese a cualquier otro impacto negativo que podrían estar ocasionando a la planta (Powell et al. 2011).

Por último, puede observarse que $K$. weberbaueri puede estar en floración en cualquier momento del año y por lo tanto tener una importante producción de semillas, aspecto trascendente para la especie porque luego de su fructificación sucede la muerte del individuo, sin embargo es necesario determinar el éxito de las semillas y su real aporte a la probabilidad del establecimiento de nuevos individuos.

\section{Agradecimientos}

Expresamos nuestra gratitud al Consejo Superior de Investigación de la Universidad Nacional Mayor de San Marcos, por el financiamiento parcial del presente estudio a través del proyecto de investigación del profesor Asunción Cano (Estudio Nº910001161). Así mismo a la Dra. Mónica Arakaki por su apoyo y al Blgo. José Roque Gamarra por la elaboración del mapa de las localidades del estudio.

\section{Literatura citada}

Arroyo J. 1988. Atributos florales y fenología de la floración en matorrales de sur de Espańa. Lagascalia 15(1): 43-78.

Brako L. \& J.L. Zarucchi. 1993. Catalogue of the Flowering Plants and Gymnosperms of Peru. Monogr.Syst. Bot. Missouri Bot. Gard. 45: i-Xl, 1-1286.

Candolle A. de. 1817. Regni Vegetabilis Systema Naturale, Paris. Vol. 1: 130, 228.

Cursach, J \& J. Rita 2012. Reproductive biology of Ranunculus weyleri (Ranunculaceae), a narrowly endemic plant form the Balearic Islands with disjunct populations. Flora 726-735. DOI http://dx.doi. org/10.1016/j.flora.2012.07.004

D’Ambrogio de Argüeso A. 1970. Manual de técnicas en histología vegetal. 1era Edición. Buenos Aires. Pp 5-79.

Lourteig A. 1956a. Ranunculáceas de Sudamérica Tropical. Memoria de la Sociedad de Ciencias Naturales La Salle 16 (43): 19-88.

Lourteig A. 1956b. Ranunculáceas de Sudamérica Tropical. Memoria de la Sociedad de Ciencias Naturales La Salle 16 (44): 125-228.

Macbride J.F. 1937.Ranunculaceae in Flora of Peru. Field Museum of Natural History; Botanical Series 13: 639-661.

Osborn, M., P. Kevan \& M. Lane. 1988. Pollination biology of Opuntia polyacantha and Opuntia plaeacantha (Cactaceae) in southern Colorado. Plant Syst. Evol. 159: 85-94. DOI 10.1007/BF00937427

Tamura M. 1993. Ranunculaceae. En: Kubitzki K., J.G. Rohwer, \& V. Bittrich (Eds.). The Families and Genera of Vascular Plants. II. Flowering Plants - Dicotyledons.Springer-Verlag: Berlín. Vol. 2: 563-583.

Timmerman-Erskine M. \& R. Boyd 1999. Reproductive biology of endangeres plant Clematis socialis (Ranunculaceae). Journal of the Torrey Botanical Society 126 (2), pp. 107-116.

Torres D.C., L.A. Cavieres, C.M. Ramirez, \& M.T.K. Arroyo, 2007. Consecuencias de las variaciones microclimaticas sobre la visita de insectos polinizadores en dos especies de Chaetanthera (Asteraceae) en los Andes de Chile central. Revista Chilena de Historia Natural 80: 455-468.

Ulloa C., J. Zarucchi \& B. León. 2004. Diez años de adiciones a la flora del Perú: Arnaldoa, Ed. Especial 7: 242. 